\title{
Trap-state suppression and improved charge transport in PbS quantum dot solar cells with synergistic mixed ligand treatments
}

Santanu Pradhan ${ }^{1}$, Alexandros Stavrinadis ${ }^{1}$, Shuchi Gupta ${ }^{1}, \mathrm{Yu} \mathrm{Bi}^{1}$, Francesco Di Stasio ${ }^{1}$, and Gerasimos Konstantatos ${ }^{1,2 *}$

${ }^{1}$ ICFO-Institut de Ciencies Fotoniques, The Barcelona Institute of Science and Technology, 08860 Castelldefels (Barcelona), Spain

${ }^{2}$ ICREA-Institució Catalana de Recerca i Estudis Avançats, Passeig Lluís Companys 23, 08010 Barcelona, Spain

* gerasimos.konstantatos@icfo.es

\begin{abstract}
The power conversion efficiency of colloidal $\mathrm{PbS}$ quantum dot based solar cells is significantly hampered by lower than expected open circuit voltage $\left(\mathrm{V}_{\mathrm{OC}}\right)$. The $\mathrm{V}_{\mathrm{OC}}$ deficit is considerably higher in QD based solar cells compared to other types of existing solar cells due to in-gap trap induced bulk recombination of photogenerated carriers. Here, we report ligand exchange procedure based on a mixture of zinc iodide and 3-mercaptopropyonic acid to reduce the $\mathrm{V}_{\mathrm{OC}}$ deficit without compromising the high current density. This layer-by-layer solid state ligand exchange treatment enhanced the photovoltaic performance from $6.62 \%$ to $9.92 \%$ with a significant improvement in $\mathrm{V}_{\mathrm{OC}}$ from $0.58 \mathrm{~V}$ to $0.66 \mathrm{~V}$. We further employed opto-electronic characterization, XPS and PL spectroscopy to understand the origin of $\mathrm{V}_{\mathrm{OC}}$ improvement. The mixed ligand treatment reduces the sub bandgap traps and significantly reduces the bulk recombination in the devices.
\end{abstract}




\section{Introduction}

In recent years, tremendous development of $\mathrm{PbS}$ colloidal quantum dots (CQDs) based photovoltaics (PV), have made this third generation PV technology promising for commercialization $^{[1]}$. Some attractive features of this technology are its spectral coverage, its compatibility with flexible substrates and with solution-based and scalable fabrication techniques. The continuous research efforts in this field have improved the solar cell power conversion efficiency (PCE) of PbS CQD cells to more than 11\% in 2016 (certified PCE $11.3 \%)^{[2]}$ from a PCE of less than $1 \%$ a decade $\mathrm{ago}^{[3]}$. This progress in device performance has been due to advanced understanding of the material properties as well as the device operation principles ${ }^{[4]}$. Understanding effects related to quantum confinement ${ }^{[5,6]}$, surface

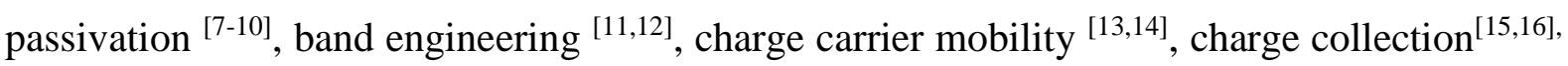
remote trap passivation ${ }^{[17,18]}$ and carrier recombination dynamics ${ }^{[19,20]}$ in CQD solids, have led to better CQD solar cells.

Despite these improvements, the PCE of these solar cells are still below the expectations with one main limiting factor being the large open-circuit voltage ( $\left.V_{O C}\right)$ deficit. The $V_{\text {OC }}$ deficit, defined as the difference between the band gap and the open circuit voltage is higher for these devices $\left(0.55 \mathrm{~V}\right.$ to $0.75 \mathrm{~V}$ for 1.1 to $1.4 \mathrm{eV}$ band gap) ${ }^{[21]}$ compared to the $\mathrm{c}-\mathrm{Si}(0.38 \mathrm{~V}$ for 1.12 eV band gap), CIGS (0.35 V for $1.1 \mathrm{eV}$ band gap), GaAs (0.3V for $1.42 \mathrm{eV}$ band gap) and organometallic halide perovskite $\left(0.4 \mathrm{~V}\right.$ for $1.5 \mathrm{eV}$ bandgap) based solar cells ${ }^{[22]}$. Though the underlying reasons for this $\mathrm{V}_{\mathrm{OC}}$ deficit in the case of $\mathrm{PbS}$ QDs solar cells are not fully understood yet, the abundance of in-gap states, introduced by the presence of unpassivated surface sites and off-stoichiometries ${ }^{[21,23]}$ of QDs, are considered the main suspects. The recent high efficiency devices using halide passivation (mainly organic iodide) show improved stability and high short-circuit current density (Jsc) around $25 \mathrm{~mA} / \mathrm{cm}^{2}$ mainly due to improved charge carrier mobility but the Voc is limited to half the bandgap of the QDs. In contrast, solar 
cell devices with QDs passivated solely with organic ligands such as EDT, BDT, and MPA have shown higher Voc, yet they suffer from low mobility, poor charge transport and hence low PCE ${ }^{[8,11,24]}$. To reduce the amount of in-gap traps, as well as to improve charge collection, simultaneous organic and inorganic ligand treatment may be necessary as has been demonstrated by Ip et. al. ${ }^{[25]}$. In that report, the halide treatment of the QDs was performed in solution and was followed by the organic ligand treatment on the QD films during the layerby-layer (LbL) growth.

Inspired by this approach we posited that a synergistic hybrid ligand treatment taking place simultaneously in-situ during the LbL growth of the films would offer a more balanced passivation of the surface of QDs and result in higher quality films. To implement this we decided to employ instead inorganic halide salts in order to circumvent the incompatibility challenges in mixing organic halide (such as TBAI or EMII) with organic ligands. Metal halides have been incorporated in the QD matrix using precursor solution during the synthesis ${ }^{[26]}$ or using post-synthetic treatments ${ }^{[27]}$. Crisp et.al. showed reported an efficiency of $7 \%$ employing a layer-by- layer solid state metal iodide ligand treatment yet these devices suffered from lower $\mathrm{V}_{\mathrm{OC}}$ and fill factor (FF) due to sub-bandgap energy states introduced through uncharged $\mathrm{Pb}$ or undesired contamination during the ligand exchange process ${ }^{[28]}$. Recently, Ko et.al. have showed that a short treatment of metal halide on the PbS QDs before the organic ligand treatment improved the $\mathrm{V}_{\mathrm{OC}}$ by nearly $100 \mathrm{mV}$ by balancing surface charge offstoichiometry, in turn reducing the mid-gap emissive trap density, yet the resultant efficiency was approximately $7.3 \%$ due to a $\mathrm{V}_{\mathrm{OC}}$ deficit of $0.72 \mathrm{~V}$ for $1.2 \mathrm{eV} \mathrm{PbS} \mathrm{QD}{ }^{[29]}$. These reports intrigued us to develop a layer by layer solid state ligand exchange method with mixed ligands comprising of metal iodide and organic acid to facilitate the advantages of both charge mobility improvement and trap state reduction. 
Here we introduce a ligand treatment where we mix an inorganic halide (zinc iodide $\left(\mathrm{ZnI}_{2}\right)$ ) with 3-mercaptopropionic acid (MPA) before applying them to the layer-by-layer ligand treatment. MPA is chosen as it helps in reducing the mid-gap states by protecting the surface from being oxidised ${ }^{[14]}$. On the other hand, $\mathrm{ZnI}_{2}$ is chosen as the metal iodide for better charge transport and it does not form solid complexes with MPA when the two are mixed in solution. The $\mathrm{PbS}$ QDs were deposited over $\mathrm{ZnO}$ QDs to form depleted heterojunction solar cell structure with the final 2 layers treated with 1,2-Ethanedithiol (EDT) for electron blocking layer (details in experimental section). Effect of the mixed ligand treatment on the device performance compared to the $\mathrm{ZnI}_{2}$ salt based treatment is studied thoroughly and discussed below.

\section{Results \& Discussions}

\section{Photovoltaic performance}

The scanning electron microscope (SEM) cross-sectional micrographs of the $\mathrm{ZnI}_{2}$ and $\mathrm{ZnI}_{2} \_\mathrm{MPA}$ mixed treated full devices are shown in Figures 1 (a) \& (b) respectively. The optimized active layer thickness is almost the same for the two devices. We exclude the MPA treated devices performance from the subsequent discussion as they suffer from low current, high series resistance and low PCE (Supporting Figure S1) and we therefore focus on the comparison of the $\mathrm{ZnI}_{2}$ and $\mathrm{ZnI}_{2} \_\mathrm{MPA}$ treated devices. The optimized device was achieved through mixing of MPA to $25 \mathrm{mmol} \mathrm{ZnI}_{2}$ in methanol solution $(0.01 \%(\mathrm{v} / \mathrm{v}))$. The effect of different $\mathrm{ZnI}_{2}$ and MPA mixing concentrations on the photovoltaic performance is summarized in supporting information (S2). The PV figures of merit for $\mathrm{ZnI}_{2}$ and $\mathrm{ZnI}_{2} \_\mathrm{MPA}$ treated devices are summarised in Table 1 . The average PCE improved from $6.34 \%$ to $9.62 \%$ when we mixed $\mathrm{ZnI}_{2}$ solution with MPA solution whereas the champion device showed an improved PCE of 9.92\% (showed in Figure 1(c)). The important factor behind the improvement of the device 
efficiency comes from the improvement of both $\mathrm{V}_{\mathrm{OC}}$ and $\mathrm{J}_{\mathrm{SC}}$ (short-circuit current density) whereas the fill factor (FF) improved slightly due to some minor improvements in series resistance (decreased from $86.78 \Omega$ to $76.94 \Omega$ ) and shunt resistance (increased from $16.39 \mathrm{k} \Omega$ to $20.30 \mathrm{k} \Omega$ ). The most notable change is observed in the $\mathrm{V}_{\mathrm{OC}}$, which improves from $0.568 \mathrm{~V}$ to $0.654 \mathrm{~V}$ with addition of MPA to the $\mathrm{ZnI}_{2}$ solution. To our knowledge, this is the highest $\mathrm{V}_{\mathrm{OC}}$ observed in a PbS QD based depleted heterojunction solar cell with such a high $\mathrm{J}_{\mathrm{SC}}$ for a $1.18 \mathrm{eV}$ band gap PbS QD. Even larger VOC values have been reached with smaller QDs: when their bandgap is tuned to $1.26 \mathrm{eV}$ the solar cell reached $0.7 \mathrm{~V} \mathrm{~V}$ OC. The $\mathrm{V}_{\text {OC }}$ dependence on QD band gap is summarized in supplementary information (S3). The results point to a reduced $\mathrm{V}_{\mathrm{OC}}$ deficit of $0.5-0.6 \mathrm{~V}$ compared to previous reports with $\mathrm{V}_{\mathrm{OC}}$ deficit of $0.6-0.8 \mathrm{~V}$ for energy band gap ranging from 1.1 to $1.4 \mathrm{eV}^{[21,30,31]}$. The $\mathrm{J}_{\mathrm{SC}}$ also exhibited a significant rise from $19.52 \mathrm{~mA} / \mathrm{cm}^{2}$ to $24.37 \mathrm{~mA} / \mathrm{cm}^{2}$ with MPA mixing reflecting also upon the external quantum efficiency (EQE) spectra with an overall improvement throughout the wavelength region and a significant rise in the near infra-red (IR) region for $\mathrm{ZnI}_{2}$ MPA treated device (Figure 1(d)). The stability of these devices was monitored over a period of 25 days from the device fabrication, left in air ambient conditions. The PCE improved for up to 5 days due to the initial enhancement of $\mathrm{J}_{\mathrm{SC}}$ and then decreased due to the constant degradation in FF (the figures of merit are summarised in supporting information (S4)). Nevertheless, the overall PCE still remain within nearly $90 \%$ of its initial value after 25 days.

\section{Intensity dependent Voc and Jsc analysis}

To understand the charge generation-recombination mechanism and device operation principle under light, we measured the dependence of $\mathrm{V}_{\mathrm{OC}}$ and $\mathrm{J}_{\mathrm{SC}}$ over an intensity range from 0.1 to 1 sun as depicted in Figure 2. The $\mathrm{J}_{\mathrm{SC}}$-suns curve shows similar intensity dependence for both $\mathrm{ZnI}_{2}$ and $\mathrm{ZnI}_{2} \_\mathrm{MPA}$ mixed ligand treated device. The curves were fitted with the relation $J_{S C}$ $\propto \Phi^{p}$, where $\Phi$ is the intensity and $\mathrm{p}$ is the exponent for the dependence. The calculated $\mathrm{p}$ factor 
from both curves shows a value of 0.96 . This implies that photocurrent in both devices is determined by the generation rate of electron-hole pairs due to photon absorption and not by space-charge region formed due to unbalanced charge transport of electrons and holes ${ }^{[32]}$. The major difference is observed in the suns- $\mathrm{V}_{\mathrm{OC}}$ dependence for $\mathrm{ZnI}_{2}$ and mixed ligand treated devices. The logarithmic fitting of this dependence yields the ideality factor ( $\boldsymbol{\eta}$ ) (supporting information S5). The fitting of the intensity dependent $\mathrm{V}_{\text {OC }}$ curve shows that $\boldsymbol{\eta}$ is reduced to 1.05 from 1.41 with the application of MPA mixing. This value of $\boldsymbol{\eta}$, approaching unity, is an exciting result, as most of the high efficiency depleted heterojunction solar cells with PbS QDs reported $\boldsymbol{\eta}$ around $1.5^{[21,33,34]}$. The value of $\boldsymbol{\eta}$ in between 1 and 2 implies the presence of ingap traps and their influence in the recombination dynamics ${ }^{[35,36]}$. The in-gap emissive states have been identified as one of the most relevant roadblock in achieving higher $\mathrm{V}_{\mathrm{OC}}$. $\boldsymbol{\eta}$ close to 1 points to reduction of in-gap trap states in the QDs with $\mathrm{ZnI}_{2} \_\mathrm{MPA}$ treatment. The cleaner band-gap results in band to band recombination and reduction in trap-mediated recombination losses.

\section{Recombination dynamics}

We further employ transient photovoltage (TPV) and photocurrent (TPC) techniques in order to gain more insights and quantitative information regarding charge transport, recombination dynamics, and trap state density. Small perturbation TPV measurements directly measure charge recombination in $\mathrm{PbS}$ QD solar cells. The lifetime $(\boldsymbol{\tau})$ calculated from the $\mathrm{V}_{\mathrm{OC}}$ decay curve with different light bias intensities is shown in Figure 3(a). The slower recombination lifetime for the $\mathrm{ZnI}_{2} \_\mathrm{MPA}$ treated device compared to $\mathrm{ZnI}_{2}$ predicts a lower recombination loss. The recombination rate $(R)$ (defined as the ratio between the excess carrier concentration with the pulse application and the recombination lifetime) plot vs $\mathrm{V}_{\mathrm{OC}}$ gives more insight about the charge recombination dynamics (see in Figure 3(b)). The $\mathrm{ZnI}_{2}$ treated device showed relatively higher $R$ even at low $\mathrm{V}_{\text {OC. }}$ Using the TPV and TPC techniques, we try to understand 
trap states in QDs according to a reported procedure ${ }^{[25]}$ (details in the supporting information S6). The result showed in Figure 3(c) confirms a cleaner in-gap trap density in case of $\mathrm{ZnI}_{2} \_\mathrm{MPA}$ treated device. This direct evidence of lower in-gap state density is consistent with the evidence of improved ideality factor and lower recombination losses.

We further plot together, in Figure 3(d), the TPC behaviour of these two devices to compare the charge transport behaviour inside the devices. The $\mathrm{ZnI}_{2} \_$MPA treated device showed a much faster photocurrent transient (transit time $0.18 \mu \mathrm{s}$ ) compared to the $\mathrm{ZnI}_{2}$ treated one (transit time $1.16 \mu \mathrm{s}$ ). This nearly 10 times acceleration in transit time indicates higher charge carrier mobility and better charge transport in $\mathrm{ZnI}_{2} \_$MPA treated QDs, consistent with the higher short-circuit current in these devices compared to $\mathrm{ZnI}_{2}$ treated ones. A rough idea of the transit mobility can be obtained considering built-in potential $\left(\mathrm{V}_{\mathrm{bi}}\right)$ as the only source of electric field assisting in the charge transport through drift ${ }^{[37]}$. $\mathrm{V}_{\mathrm{bi}}$ of the devices was obtained from the Mott-Schottky plot of the capacitance voltage characteristics (Supporting information S7). $\mathrm{V}_{\text {bi }}$ was found to be $0.72 \mathrm{~V}$ in $\mathrm{ZnI}_{2} \_$MPA treated device compared to $0.61 \mathrm{~V}$ in case of $\mathrm{ZnI}_{2}$ treated device. The calculated transit mobility was found to be increased to $3.67 \times 10^{-3} \mathrm{~cm}^{2} \mathrm{~s}^{-1} \mathrm{~V}^{-}$ ${ }^{1}$ with the addition of MPA in the ligand from $6.84 \times 10^{-4} \mathrm{~cm}^{2} \mathrm{~s}^{-1} \mathrm{~V}^{-1}$. This almost 5 times improvement in mobility further corroborates the improved surface passivation enabled via the hybrid ligand passivation over the $\mathrm{ZnI}_{2}$-only device.

\section{X-ray photoelectron spectroscopy analysis}

We further employ X-ray photoelectron spectroscopy (XPS) to correlate the physico-chemical effects of the ligand treatment on the $\mathrm{PbS}$ surface with the device performance and optoelectronic properties. Figure 4 shows analyzed high resolution S2p, O1s and Pb4f XPS spectra of MPA, $\mathrm{ZnI}_{2}$ and $\mathrm{ZnI}_{2} \_\mathrm{MPA}$ treated PbS QDs. Similarly to different previous reports $[8,23,38,39]$, these spectra are deconvoluted by considering $\mathrm{S}$ attributed to $\mathrm{PbS}$, bound and 
unbound thiolates, $\mathrm{Pb}$ attributed to undercharged $\mathrm{Pb}, \mathrm{Pb}-\mathrm{S}$ and $\mathrm{Pb}$-carboxylate, and $\mathrm{O}$ attributed to $\mathrm{Pb}-\mathrm{O},-\mathrm{OH}, \mathrm{H} 2 \mathrm{O}$ and $-\mathrm{COO}-$.The $\mathrm{S} 2 \mathrm{p}$ spectra show increased contents of bound thiolates and suppressed presence of unbound thiolates when the hybrid $\mathrm{ZnI}_{2} \_$MPA treatment is employed. The reduction of dangling thiolates signifies better surface passivation with mixed ligand treatment. For $\mathrm{O} 1 \mathrm{~s}$ spectra, $\mathrm{ZnI}_{2}$ treated $\mathrm{QDs}$ shows presence of atomic $\mathrm{PbO}$ along with $-\mathrm{OH}$ which introduce sub-bandgap states. With MPA treatment, the amount of atomic $\mathrm{O}$ diminished but the presence of $-\mathrm{OH}$ increased a lot. Cao et. al. showed device performance degradation that is correlated with $-\mathrm{OH}$ presence ${ }^{[8]} . \mathrm{ZnI}_{2} \_\mathrm{MPA}$ treated dots showed reduction of both atomic $\mathrm{O}$ and $-\mathrm{OH}$ from the surface, species that are associated with the formation of traps in $\mathrm{PbS}$ QDs. Further, the reduction of carboxylate- $\mathrm{Pb}$ and metallic $\mathrm{Pb}$ in mixed ligand treated device compared to the $\mathrm{ZnI}_{2}$ treated and MPA treated devices endorse the fact of reduction of emissive sub-band gap states as with $\mathrm{ZnI}_{2}$ treated device undercharged $\mathrm{Pb}$ leads to the formation of emissive sub-band gap ${ }^{[23]}$. We summarize the quantitative analysis of the XPS spectra to understand the stoichiometry of the ligand treated devices in Table 1 . The overall contamination of $\mathrm{Zn}, \mathrm{O}, \mathrm{C}$ are much less in $\mathrm{ZnI}_{2} \_\mathrm{MPA}$ treated devices compared to the $\mathrm{ZnI}_{2}$ treated device. We attribute the presence of zinc in the films to the formation of undesired organometallic zinc complexes, most likely zinc oleate, and not to the presence of $\mathrm{ZnI}_{2}$. This can be further understood by examining the $\mathrm{Zn}$ and I ratio of the $\mathrm{ZnI}_{2}$ and $\mathrm{ZnI}_{2}$ MPA treated devices. In both $\mathrm{ZnI}_{2}$ and $\mathrm{ZnI}_{2}$ MPA treated samples, the $\mathrm{I} / \mathrm{Zn}$ ratio is not 2 which would be expected if zinc is attributed to unreacted $\mathrm{ZnI}_{2}$. In fact, for the $\mathrm{ZnI}_{2}$ treated sample, excess $\mathrm{Zn}$ is observed whereas for $\mathrm{ZnI}_{2} \_\mathrm{MPA}$ treated device, $\mathrm{I} / \mathrm{Zn}$ ratio is higher than 2 . Therefore, we propose that upon treating the films with $\mathrm{ZnI}_{2}$, iodide is exchanged with oleate on the surface of the dots and that leads to the formation organometallic zinc complexes onto the QD films. Washing away these zinc containing impurities is greatly facilitated by the presence of MPA. This hypothesis is further corroborated by the fact that the $\mathrm{Zn} 2 \mathrm{p} 3 / 2$ peak (supporting Figure 
S6) shifts $0.3 \mathrm{eV}$ for $\mathrm{ZnI}_{2} \_\mathrm{MPA}$ treated sample compared to $\mathrm{ZnI}_{2}$ treated samples. Overall, the suppression of unwanted chemical species from the QD surface upon our mixed ligand treatment, can explain the improved optoelectronic properties and performance of the as made QD films and devices.

\section{Photoluminescence studies}

We employ photoluminescence (PL) spectroscopy to further support our claim about the reduction of emissive in-gap states with $\mathrm{ZnI}_{2}$ MPA treated device. The unpassivated sites induce mid-gap states which in turn affect the PL spectra as they introduce different channels of radiative and non-radiative recombination. Figure 5(a) compares the emission intensity of $\mathrm{ZnI}_{2}$ and $\mathrm{ZnI}_{2} \_$MPA treated mixed ligand treated PbS QDs. Massive improvement of the bandedge PL intensity was observed when QDs were treated with mixed ligand compared to only $\mathrm{ZnI}_{2}$ treated ones, similar to MPA treated samples (supporting Figure S7). This suggests that with mixed ligand treatment the non-radiative emission channels are partially suppressed compared to $\mathrm{ZnI}_{2}$ treated QDs. Moreover, strong emission corresponding to $0.84 \mathrm{eV}$ with $\mathrm{ZnI}_{2}$ treated QDs was observed (Figure 5(b)). This sub-band gap emission is a clear evidence of the presence of poorly emissive in-gap states strongly present in the case of $\mathrm{ZnI}_{2}$ treated films. This sub-band gap emission was drastically supressed with the addition of MPA. The PL spectra also suggests that $\mathrm{ZnI} 2$ treated dots have higher Urbach tail $\left(\mathrm{E}_{\mathrm{U}}\right)$ energies compared to mixed ligand treated ones. To verify this, we have calculated $\mathrm{E}_{\mathrm{U}}$ from the corresponding slope of $\mathrm{EQE}$ spectra of the devices as described by Hages et. al. ${ }^{[40]}$. The calculated $\mathrm{E}_{\mathrm{U}}$ reduced to $23 \mathrm{meV}$ for mixed ligand treated device compared to $45 \mathrm{meV}$ of the $\mathrm{ZnI}_{2}$ passivated device as shown in Figure 5(c). Likewise, the analytic calculation of the corresponding ideality factor ${ }^{[41]}$ predicts similar kind of difference in $\mathrm{E}_{\mathrm{U}}$ (Supporting information $\mathrm{S} 10$ ). Importantly the $\mathrm{E}_{\mathrm{U}}$ is lower than room temperature thermal energy $(26 \mathrm{meV})$ with mixed ligand treatment. This leads 
to enhanced thermal de-trapping of minority carriers and thereby reduced trap assisted recombination.

\section{Conclusions}

We have shown that a mixed ligand treatment comprising hybrid inorganic metal iodide salts $\left(\mathrm{ZnI}_{2}\right)$ and organic MPA improves drastically the PV performance in PbS QD solar cells. This approach exploits the complementary benefits of inorganic ligands for improved carrier transport and robustness with those of organic ligands for improved passivation and in-gap state suppression. The synergy of the two leads to the simultaneous achievement of high Voc and Jsc and an overall PCE of nearly 10\%. To elucidate the origins of the synergistic mechanisms at play we have employed a very broad range of characterization techniques to show that the hybrid passivation suppresses dramatically the presence of in-gap states and the associated recombination mechanisms thereof and has therefore led to higher performance solar cells compared to those based on either of the two ligand treatments. On a broader perspective this work paves the way towards new hybrid ligand engineering of QD solids as means to reach record performance QD solar cells.

\section{Experimental Section}

Synthesis of PbS QDs: Schlenk technique was used to synthesize PbS QDs following standard recipe. 2 mmol lead oxide (PbO), $4.7 \mathrm{mmol}$ oleic acid, and 9.4 mmol 1-octadecene (ODE) were pumped overnight at $95{ }^{\circ} \mathrm{C}$. Then $15 \mathrm{~mL}$ of ODE was added and the temperature of the reaction was raised to $120^{\circ} \mathrm{C}$. When this point is reached, $1 \mathrm{mmol}$ hexamethyldisilathiane mixed with $10 \mathrm{~mL}$ ODE was quickly injected. The heating was stopped (without removing the heating mantle) and the sample allowed to cool down slowly ( $\sim 1$ hour). The NCs were isolated by adding acetone and then centrifuging, purified by dispersion/precipitation with toluene/acetone 
3 times, and finally dispersed in anhydrous toluene. The obtained QDs were further treated with n-butylamine ligand for 1 day in the glove box and then cleaned with methanol. The final QDs were dispersed in anhydrous toluene $\left(30 \mathrm{mg} \mathrm{ml}^{-1}\right.$ ) before applying for the device formation.

Photovoltaic device fabrication: ITO coated glass substrates were cleaned thoroughly with soap water and acetone respectively before boiling in 2-propanol for $10 \mathrm{~min}$. Then the substrates were dried using nitrogen gun prior to material deposition. $\mathrm{ZnO}$ nanocrystals were prepared using the standard method reported elsewhere ${ }^{[17]}$. The concentration of the prepared $\mathrm{ZnO}$ nanocrystal colloidal solution in chloroform was fixed at $45 \mathrm{mg} \mathrm{ml}^{-1}$. For $\mathrm{ZnO}$ bottom layer formation, the cleaned ITO coated substrates were fully covered with $\mathrm{ZnO}$ nanocrystal solution and were immediately spun at $3500 \mathrm{rpm}$ for $30 \mathrm{sec}$. The substrates were then shifted to a hotplate with pre-set temperature of $250{ }^{\circ} \mathrm{C}$ and left for 30 min to bake. After that, the heat source was switched off and the substrates were allowed to cool down to room temperature. The active layer of the device was formed by sequential layer by layer spin coating technique. For a single layer, cleaned $\mathrm{PbS}$ solution in anhydrous toluene (30 mg ml-1 concentration) was employed on the $\mathrm{ZnO}$ covered substrate and spun immediately with $2500 \mathrm{rpm}$ for $15 \mathrm{sec}$. Then the rotation was stopped before applying 5 drops of $\mathrm{ZnI}_{2}$ (25 mM in methanol) or $\mathrm{ZnI}_{2} \_$MPA mixed ligand (25 $\mathrm{mM} \mathrm{ZnI}_{2}$ with $0.01 \% \mathrm{MPA}(\mathrm{v} / \mathrm{v})$ in Methanol) solution to the PbS layer and waited $5 \mathrm{sec}$ for the ligand exchange. After that, the rotation was started immediately to dry the sample for 10sec. Then the unreacted ligands were washed twice with few drops of methanol solution and the film was dried with $10 \mathrm{sec}$ more rotation. The process was repeated for 12 times to get a thicker film. The final 2 layers were formed with EDT treatment. The PbS layer was treated with $0.02 \%$ EDT in acetonitrile for $30 \mathrm{sec}$ and then was rotated for $10 \mathrm{sec}$ to dry it before washed it twice with few drops of acetonitrile solution. The as synthesized films were kept in the glovebox for overnight prior to the metal electrode deposition. Au metal was 
deposited in a Kurt J. Leskar Nano 36 system at a rate of $2 \AA \mathrm{S}^{-1}$ for the final thickness of 150 $\mathrm{nm}$. The active device area was determined by a circular shadow mask of $2 \mathrm{~mm}$ diameter. After the electrode deposition, the devices were transferred to glovebox to anneal at $80^{\circ} \mathrm{C}$ on a preheated hotplate for 5 minutes. The devices were taken out of the glovebox and stored in air for further characterizations.

Photovoltaic performance characterization: All the PV characterizations were performed in ambient conditions. The device I-V responses were performed using a Keithley 2400 source meter. Illumination intensity of AM 1.5 was maintained using a class AAA solar simulator (Oriel sol3A, Newport Corporation). The accuracy of the measurement was determined as $\pm 4 \%$.

EQE measurements: EQE measurements were performed with an in-house build experimental set-up by using chopped $(220 \mathrm{~Hz}$, Thorlab) monochromatic illumination. The power was measured with a calibrated Newport-UV power meter. The device response of the chopped signal was measured using a Stanford Research system lock-in amplifier (SR830) which was fed by a Stanford Research system low noise current pre-amplifier (SR570). The final EQE spectra were obtained with the help of LabVIEW program.

Intensity dependent $V_{O C}$ and $J_{S C}$ measurements: The intensity depended $\mathrm{V}_{\mathrm{OC}}$ and $\mathrm{J}_{\mathrm{SC}}$ were measured with a LED lamp calibrated with Newport 818-UV power meter and an Agilent 4000X oscilloscope. The intensity of the lamp was varied with the DC applied bias from the function generator of the oscillator. The voltage was recorded on the oscilloscope with input impedance $1 \mathrm{M} \Omega$ and the current with $50 \Omega$. The accuracy of this measurement was estimated to be within $\pm 8 \%$.

Transient photovoltage and photocurrent techniques: Transient photovoltage (TPV) and photocurrent (TPC) of the devices were measured with an in-house-built set-up. The set-up 
comprises a LED lamp to provide steady state white bias light, a $637 \mathrm{~nm}$ wavelength laser (Vortran Stradus) and an Agilent 4000X oscilloscope. The LED lamp was used to get steady $\mathrm{V}_{\mathrm{OC}}$ of the device. The intensity of the lamp was controlled by the external applied DC bias and was attenuated with metal-mesh according to the necessity. The laser was controlled by the function generator with a frequency of $10 \mathrm{~Hz}$ and pulse width of $100 \mu$ s. The intensity of the laser was controlled to keep the voltage transient amplitude under 5\% of the steady state light bias. The oscilloscope records the data using $1 \mathrm{M} \Omega$ input impedance for the TPV measurement and $50 \Omega$ for TPC measurement. The $\mathrm{V}_{\mathrm{OC}}$ decay curves were fitted with exponential decay to find the recombination time. The TPC curve was integrated to get the charge generated $(\Delta \mathrm{Q})$ in the devices due to the laser pulse. The capacitance $(\mathrm{C})$ was calculated from the $\mathrm{C}=\Delta \mathrm{Q} / \Delta \mathrm{V}_{\mathrm{OC}}$ relation. The total charge carrier was calculated from the integration of C vs VoC plot. Charge carrier density was calculated by dividing the total charge carriers with the device volume.

X-ray photoelectron spectroscopy measurements: XPS experiments were performed in a PHI 5500 Multitechnique System with a monochromatic X-ray source (Aluminium $\mathrm{K}_{\alpha}$ line of $1486.6 \mathrm{eV}$ energy and $350 \mathrm{~W}$ power), placed perpendicular to the analyser axis and calibrated using the $3 \mathrm{~d} 5 / 2$ line of $\mathrm{Ag}$ with a full width at half maximum (FWHM) of $0.8 \mathrm{eV}$. All measurements were performed in ultra-high vacuum chamber with pressure between $5 \times 10^{-9}$ and $2 \times 10^{-8}$ torr. The samples were prepared with layer-by-layer method described above and each of them are with 5 layers. The collected data were processed with XPSPEAK41 software for quantitative analysis. The deconvolution of the XPS spectra was performed by fitting a sum of Lorentzian-Gaussian functions (always with $80 \%$ Gaussian weighting) to the experimental data. For consistency, the full-width-at half-maximum (FWHM) of all Gaussian-Lorentzian functions for the same spectral regions and peaks of different samples was kept the same. 
Photoluminescence measurements: Photoluminescence measurements were carried out using a Horiba Jobin Yvon iHR550 Fluorolog system along with a Hamamatsu R5509-73 photomultiplier tube detector. Samples were excited with a $637 \mathrm{~nm}$ laser (Vortran Stradus). The acquired raw data was processed first subtracting the dark counts and normalised with respect to the absorption peak of the corresponding films.

\section{Acknowledgements}

We acknowledge financial support from the European Research Council (ERC) under the European Union's Horizon 2020 research and innovation programme (grant agreement No 725165), the Spanish Ministry of Economy and Competitiveness (MINECO) and the "Fondo Europeo de Desarrollo Regional" (FEDER) through grant MAT2014-56210-R. This work was also supported by AGAUR under the SGR grant (2014SGR1548) and by the EC under the Graphene Flagship (contract no. CNECT-ICT-604391). We also acknowledge financial support from Fundacio Privada Cellex and from the Spanish Ministry of Economy and Competitiveness, through the "Severo Ochoa" Programme for Centres of Excellence in R\&D (SEV-2015-0522). F.D.S. acknowledges support from the Marie Curie Standard European Fellowship "NANOPTO" (H2020-MSCA-IF-2015-703018) 


\section{References}

(1) E. H. Sargent, Nat. Photonics, 2012, 6, 133.

(2) M. Liu, O. Voznyy, R. Sabatini, F. P. G. de Arquer, R. Munir, A. H. Balawi, X. Lan, F. Fan, G. Walters, A. R. Kirmani, S. Hoogland, F. Laquai, A. Amassian, E. H. Sargent, Nat. Mater. 2016 (DOI: 10.1038/nmat4800).

(3) S. a McDonald, G. Konstantatos, S. Zhang; P.W. Cyr, E.J.D. Klem, L. Levina, E.H. Sargent, Nat. Mater. 2005, 4, 138.

(4) G.H. Carey, A.L. Abdelhady, Z. Ning, S.M. Thon, O.M. Bakr, E.H. Sargent, Chem. Rev. 2015, 115, 12732.

(5) D.V. Talapin, C.B. Murray, Science 2005, 310, 86.

(6) P.R. Brown, D. Kim, R.R. Lunt, N. Zhao, M.G. Bawendi, J.C. Grossman, V. Bulovic ACS Nano 2014, 8, 5863.

(7) G. H. Carey, L. Levina, R. Comin, O. Voznyy, E. H. Sargent, Adv. Mater., 2015, 27, 3325 .

(8) Y. Cao, A. Stavrinadis, T. Lasanta, D. So, G. Konstantatos, Nat. Energy, 2016, 1, 16035.

(9) X. Lan, O. Voznyy, A. Kiani, F. P. G. Arquer, A. S. Abbas, G.-H. Kim, M. Liu, Z. Yang, G. Walters, J. Xu, M. Yuan, Z. Ning, F. Fan, P. Kanjanaboos, I. Kramer, D. Zhitomirsky, P. Lee, A. Perelgut, S. Hoogland, E. H. Sargent, Adv. Mater., 2016, 28, 299.

(10) S.M. Thon, A.H. Ip, O. Voznyy, L. Levina, K.W. Kemp, G.H. Carey, S. Masala, E.H. Sargent ACS nano, 2013, 7, 7680.

(11) C.-H. M. Chuang, P.R. Brown, V. Bulovic, M.G. Bawendi, Nat. Mater, 2014, 13, 796.

(12) Z. Ning, O. Voznyy, J. Pan, S. Hoogland, V. Adinolfi, J. Xu, M. Li, A. R. Kirmani,J.P. Sun, J. Minor, $\quad$ K. W. Kemp, H. Dong, L. Rollny, A. Labelle, G. Carey, $\quad$ B. Sutherland, I. Hill, A. Amassian, H. Liu, J. Tang, $\quad$ O. M. Bakr, E. H. Sargent, Nat. Mater., 2014, 13, 822.

(13) Y. Liu, M. Gibbs, J. Puthussery, S. Gaik, R. Ihly, H. W. Hillhouse, M. Law, Nano Lett. 2010, 10, 1960.

(14) K. S. Jeong, J. Tang, H. Liu, J. Kim, A. W. Schaefer, K. Kemp, L. Levina, X. Wang, S. Hoogland, R. Debnath, L. Brzozowski, E. H. Sargent, J. B. Asbury, ACS Nano, 2012, 6, 89. 
(15) X. Lan, S. Masala, E.H. Sargent, Nat. Mater., 2014, 13, 233.

(16) Z. Jin, M. Yuan, H. Li, H. Yang, Q. Zhou, H. Liu, X. Lan, M. Liu, J. Wang, E. H. Sargent, Y. Li, Adv. Func. Mater., 2016, 26, 5284.

(17) A. K. Rath, M. Bernechea, L. Martnez, F. P. G. Arquer, J. Osmond, G. Konstantatos, Nat. Photonics, 2012, 6, 529.

(18) A. K. Rath, F.P.G.Arquer, A. Stavrinadis, T. Lasanta, M. Bernechea, S.L. Diedenhofen, G. Konstantatos, Adv. Mater, 2014, 26, 4741.

(19) M. Graetzel, R. A. J. Janssen, D. B. Mitzi, E. H. Sargent, Nature, 2012, 488, 304.

(20) M. Yuan, O. Voznyy, D. Zhitomirsky, P. Kanjanaboos, E.H. Sargent, Adv. Mater., 2015, $27,917$.

(21) C.-H. M. Chuang, A. Maurano, R. E. Brandt, G. W. Hwang, J. Jean, T. Buonassisi, V. Bulovic, M.G. Bawendi, Nano Lett., 2015, 15, 3286.

(22) M. A. Green, K. Emery, Y. Hishikawa, W. Warta, E. D. Dunlop, Prog. Photovoltaics, 2016, 24, 905

(23) G. W. Hwang, D. Kim, J. M. Cordero, M. W. B. Wilson, C.-H. M. Chuang, J. C. Grossman, M. G. Bawendi, Adv. Mater, 2015, 27, 4481.

(24) Z. Ning, Y. Ren, S. Hoogland, O. Voznyy, L. Levina, P. Stadler, X. Lan, D. Zhitomirsky, E. H. Sargent, Adv. Mater., 2012, 24, 6295.

(25) A. H. Ip, S. M. Thon, S. Hoogland, O. Voznyy, D. Zhitomirsky, R. Debnath, L. Levina, L. R. Rollny, G. H. Carey, A. Fischer, K. W. Kemp, I. J. Kramer, Z. Ning, A. J. Labelle, K. W. Chou, A. Amassian, E. H. Sargent, Nat. Nanotechnol., 2012, 7, 577.

(26) J. Zhang, J. Gao, E. M. Miller, J. M. Luther, M. C. Beard, ACS Nano, 2013, 8, 614.

(27) D. Zhitomirsky, O. Voznyy, L. Levina, S. Hoogland, K. W. Kemp, A. H. Ip, S. M. Thon, E. H. Sargent, Nat. Coumn., 2014, 5, 3803.

(28) R.W. Crisp, D. M. Kroupa, A. R. Marshall, E.M. Miller, J. Zhang, M. C. Beard, J. M. Luther, Sci. Rep, 2015, 5, 9945.

(29) D.-K. Ko, A. Maurano, S. K. Suh, D. Kim, G. W. Hwang, J. C. Grossman, V. Bulovic, M. G. Bawendi, ACS Nano, 2016, 10, 3382. 
(30) W. Yoon, J.E. Boercker, M.P. Lumb, D. Placencia, E.E. Foos, J.G. Tischler, Sci. Rep., 2013, 3, 2225.

(31) A. G. Pattantyus-Abraham, I. J. Kramer, A. R. Barkhouse, X. Wang, G. Konstantatos, R. Debnath, L. Levina, I. Raabe, M. K. Nazeeruddin, M. Gratzel, E. H. Sargent, ACS Nano, 2010, 4, 3374 .

(32) N. Zhao, T. P. Osedach, L.-Y. Chang, S. M. Geyer, D. Wanger, M. T. Binda, A. C. Arango, M. G. Bawendi, V. Bulovic, ACS Nano, 2010, 4, 3743.

(33) D. Bozyigit, V. Wood, J. Mater. Chem. C, 2014, 2, 3172.

(34) Z. Sun, G. Sitbon, T. Pons, A. A. Bakulin, Z. Chena, Sci. Rep., 2015, 5, 10626.

(35) T. Kirchartz, F. Deledalle, P. S. Tuladhar, J. R. Durrant, J. Nelson, J. Phys. Chem. Lett. 2013, 4, 2371.

(36) V. Malgras, G. Zhang, A. Nattestad, T. M. Clarke, A. J. Mozer, Y. Yamauchi, J. H. Kim, ACS Appl. Mater. Interfaces, 2015, 7, 26455.

(37) Y. Shao, Z. Xiao, C. Bi, Y. Yuan, J. Huang, Nat. Comun., 2014, 5, 5784.

(38) J. M. Luther, M. Law, Q. Song, C. L. Perkins, M. C. Beard, A. J. Nozik, ACS Nano, 2008, 2, 271.

(39) V. Malgras, A. Nattestad, Y. Yamauchi, S. X. Doua, J. H. Kim, Nanoscale, 2015, 7, 5706.

(40) C. J. Hages, N. J. Carter, R. Agrawal, J. Appl. Phys. 2016, 119, 014505.

(41) T. Kirchartz, J. Nelson, Phys. Rev. B, 2012, 86, 165201. 
Tables

\begin{tabular}{ccccccc}
\hline Devices & Voc $_{\text {ov }}(\mathbf{V})$ & $\begin{array}{c}\mathbf{J S S C}_{\mathbf{s C}} \\
\left(\mathbf{m A} / \mathbf{c m}^{\mathbf{2}}\right)\end{array}$ & $\mathbf{F F}$ & $\begin{array}{c}\text { Efficiency } \\
(\boldsymbol{\%})\end{array}$ & $\mathbf{R}_{\mathbf{s}}(\boldsymbol{\Omega})$ & $\mathbf{R}_{\mathbf{s h}}(\mathbf{k} \boldsymbol{\Omega})$ \\
\hline $\mathbf{Z n I}_{2}$ & $0.568 \pm 0.006$ & $19.52 \pm 0.30$ & $0.572 \pm 0.010$ & $6.34 \pm 0.16$ & $86.78 \pm 5.01$ & $16.39 \pm 3.16$ \\
treated & $(0.58)$ & $(19.7)$ & $(0.58)$ & $(6.62)$ & & \\
\hline ZnI2_MPA & $0.654 \pm 0.005$ & $24.37 \pm 0.18$ & $0.603 \pm 0.007$ & $9.62 \pm 0.23$ & $76.94 \pm 3.57$ & $20.30 \pm 2.69$ \\
treated & $(0.66)$ & $(24.48)$ & $(0.613)$ & $(9.92)$ & & \\
\hline
\end{tabular}

Table 1: Summary of photovoltaic figures of merit for $\mathrm{ZnI}_{2}$ and mixed ligand treatments. The values in brackets indicate champion device. 


\begin{tabular}{|c|c|c|c|c|}
\hline Elements & Species & MPA & $\mathrm{ZnI}_{2}$ & ZnI2_MPA \\
\hline \multirow[t]{4}{*}{$\overline{\mathbf{P b}}$} & total & 1.00 & 1.00 & 1.00 \\
\hline & $\mathrm{Pb}-\mathrm{S}$ & 0.85 & 0.85 & 0.88 \\
\hline & $\mathrm{COO}: \mathrm{Pb}$ & 0.13 & 0.09 & 0.09 \\
\hline & Metallic $\mathrm{Pb}$ & 0.02 & 0.06 & 0.03 \\
\hline \multirow[t]{4}{*}{$\mathbf{S}$} & total & 1.02 & 0.54 & 0.87 \\
\hline & S_PbS & 0.60 & 0.54 & 0.59 \\
\hline & S_bound thiol & 0.13 & 0.00 & 0.23 \\
\hline & S_unbound thiol & 0.29 & 0.00 & 0.05 \\
\hline \multirow[t]{5}{*}{$\mathbf{O}$} & total & 0.78 & 2.36 & 0.79 \\
\hline & O_PbO & 0.00 & 0.21 & 0.00 \\
\hline & O_OH & 0.70 & 0.60 & 0.08 \\
\hline & $\mathrm{O} \_\mathrm{COOH}$ & 0.08 & 1.41 & 0.61 \\
\hline & $\mathrm{O} \_\mathrm{H}_{2} \mathrm{O}$ & 0.00 & 0.16 & 0.1 \\
\hline $\mathbf{C}$ & & 3.91 & 10.29 & 4.19 \\
\hline I & & 0.00 & 0.96 & 0.83 \\
\hline $\mathbf{Z n}$ & & 0.00 & 1.27 & 0.31 \\
\hline
\end{tabular}

Table 2: Stoichiometry of MPA, $\mathrm{ZnI}_{2}$, and $\mathrm{ZnI}_{2}$ MPA mixed ligand treated samples, based on quantitative analysis of $\mathrm{S} 2 \mathrm{p}, \mathrm{Pb} 4 \mathrm{f}, \mathrm{I} 3 \mathrm{~d}, \mathrm{Zn} 2 \mathrm{p} 3 / 2, \mathrm{O} 1 \mathrm{~s}, \mathrm{C} 1 \mathrm{~s}$ XPS spectra. 


\section{Figures}

(a)

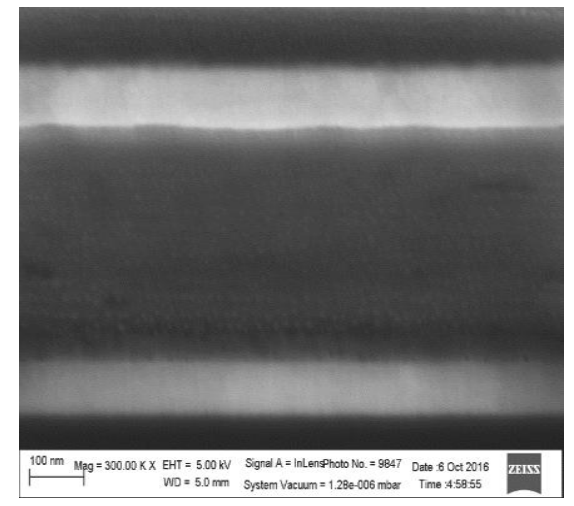

(b)

Au

\section{EDT treated PbS}

$\mathrm{ZnI}_{2} / \mathrm{MPA}$ treated

PbS

ZnO

ITO coated glass

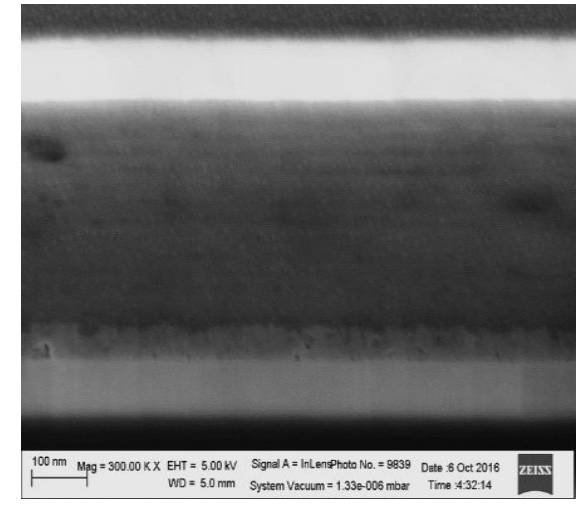

(c)

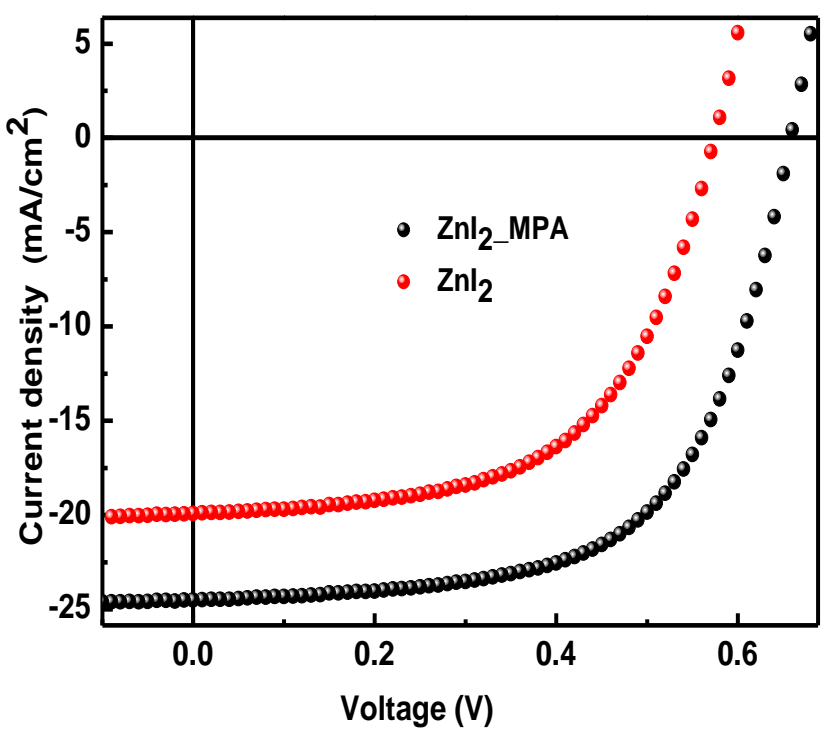

(d)

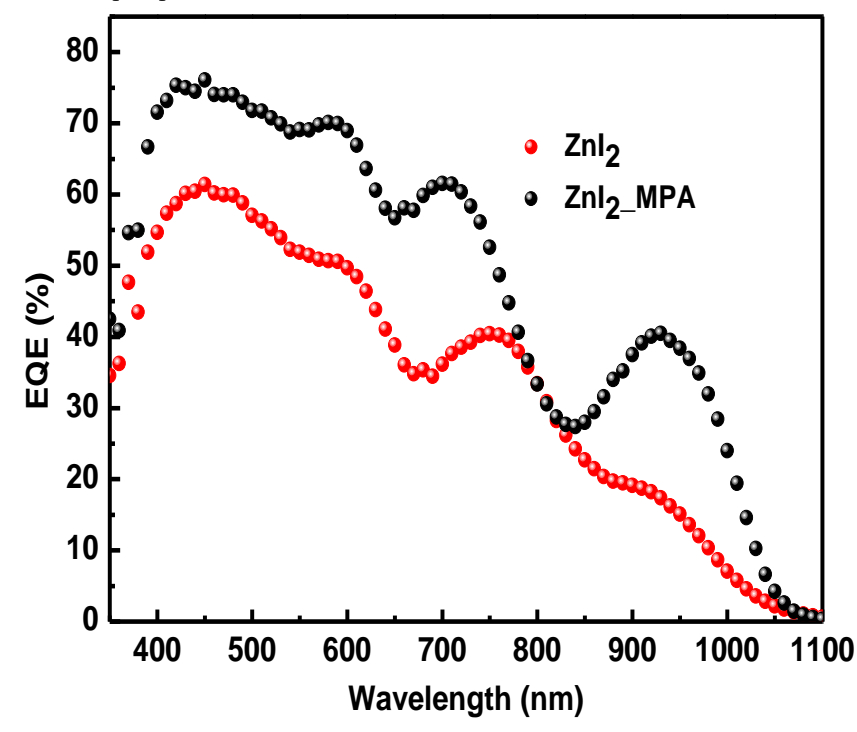

Figure 1: FIB cross-sectional images of (a) $\mathrm{ZnI}_{2}$ treated and (b) $\mathrm{ZnI}_{2} \_$MPA treated PbS QD device. (c) Current density-voltage characterizations for the PV devices to calculate efficiency. (d) EQE spectra of the $\mathrm{ZnI}_{2}$ and $\mathrm{ZnI}_{2}$ mixed with MPA treated devices. 
(a)

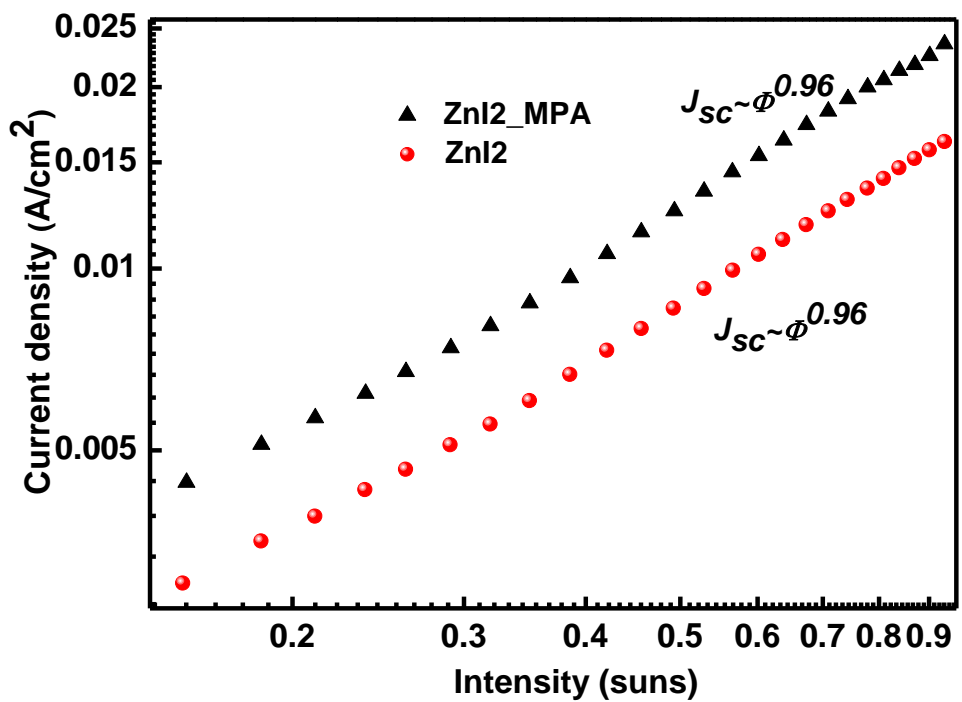

(b)

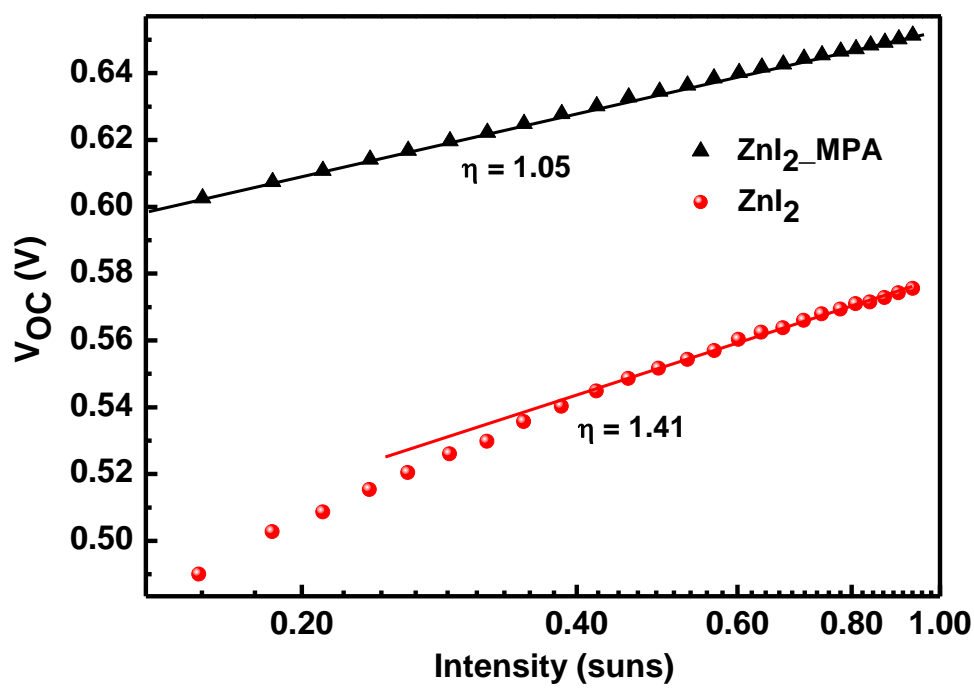

Figure 2: Intensity dependent (a) current density and (b) $\mathrm{V}_{\mathrm{OC}}$ variation for $\mathrm{ZnI}_{2}$ and mixed ligand treated PV devices. 
(a)
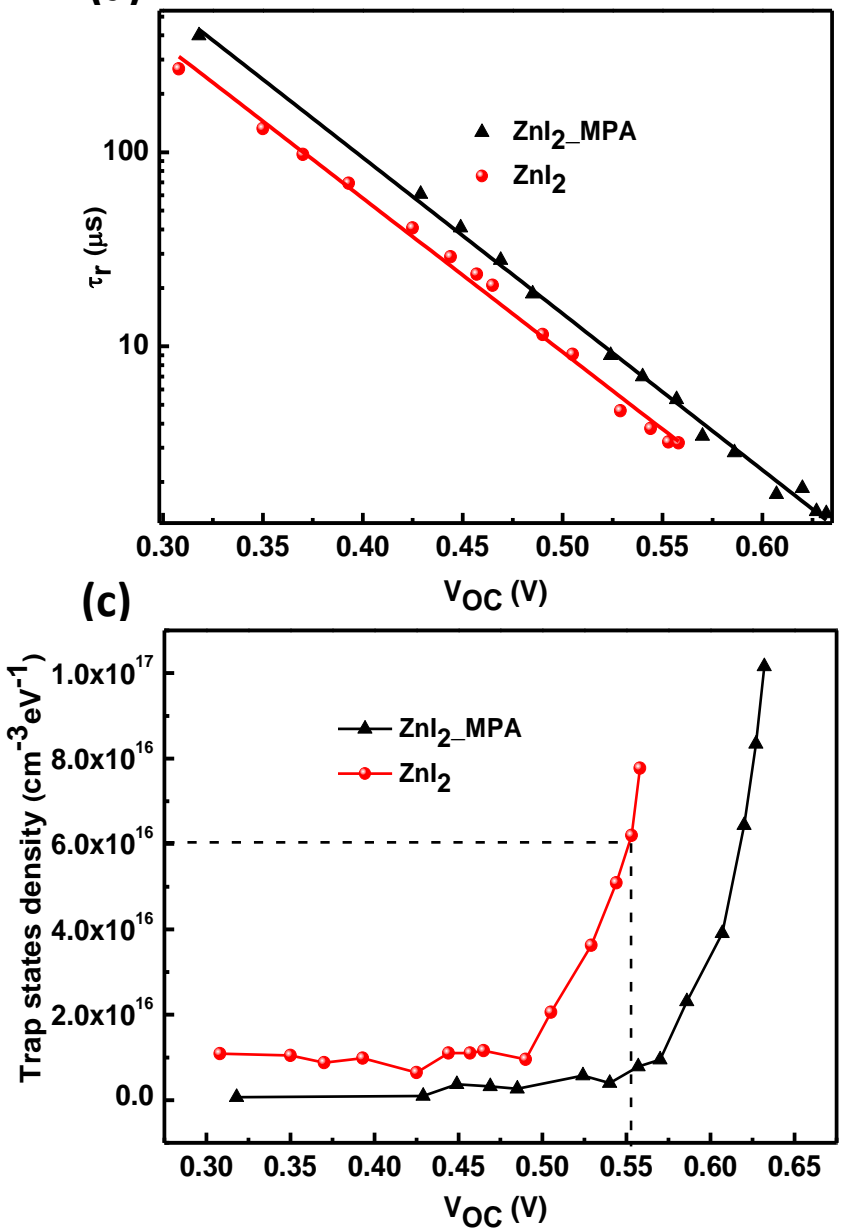

(b)

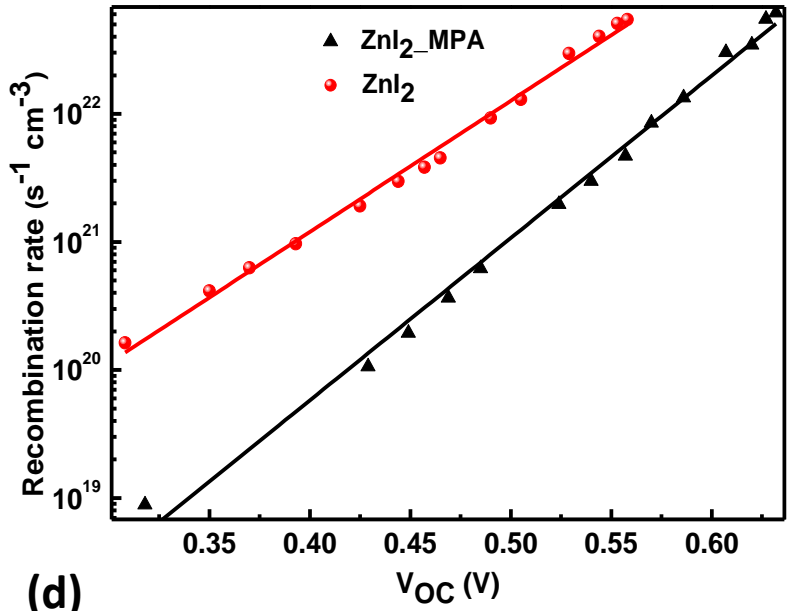

(d)

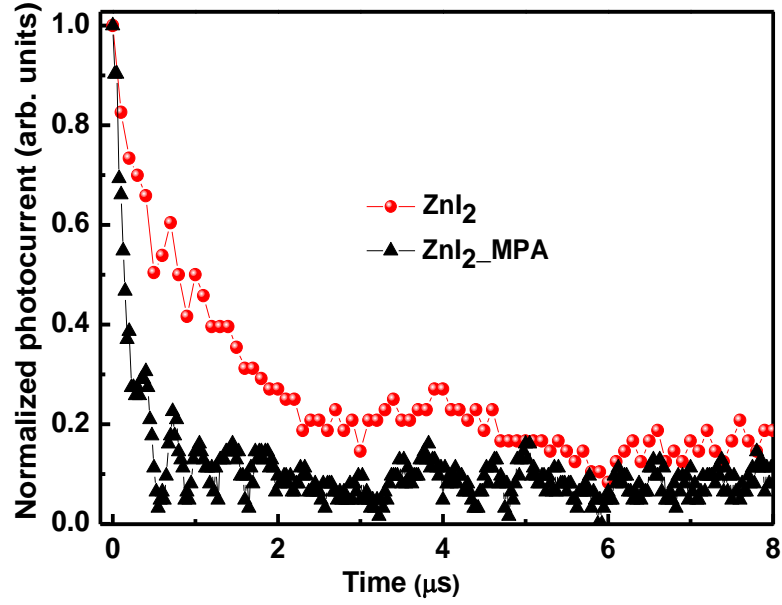

Figure 3: Comparison of the (a) recombination lifetimes, (b) recombination rate, (c) mid-gap trap density, (d) photo-current transient at short-circuit condition of $\mathrm{ZnI}_{2}$ and $\mathrm{ZnI}_{2} \_$MPA mixed ligand treated devices. All the comparisons shows the reduction of mid-gap trap states and improvement of recombination loss for $\mathrm{ZnI}_{2} \_\mathrm{MPA}$ treated device. 
(a)

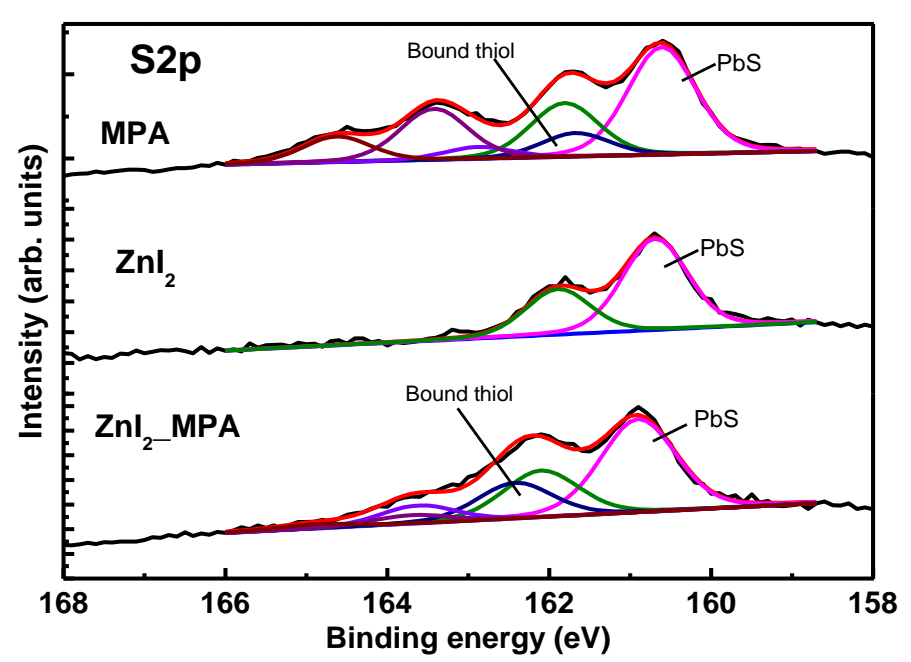

(b)

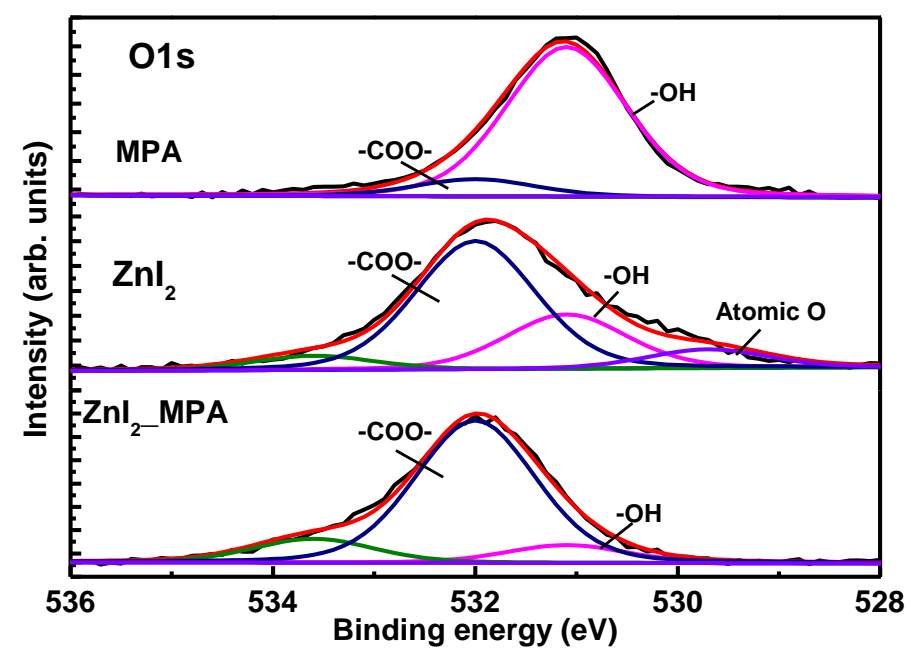

(c)

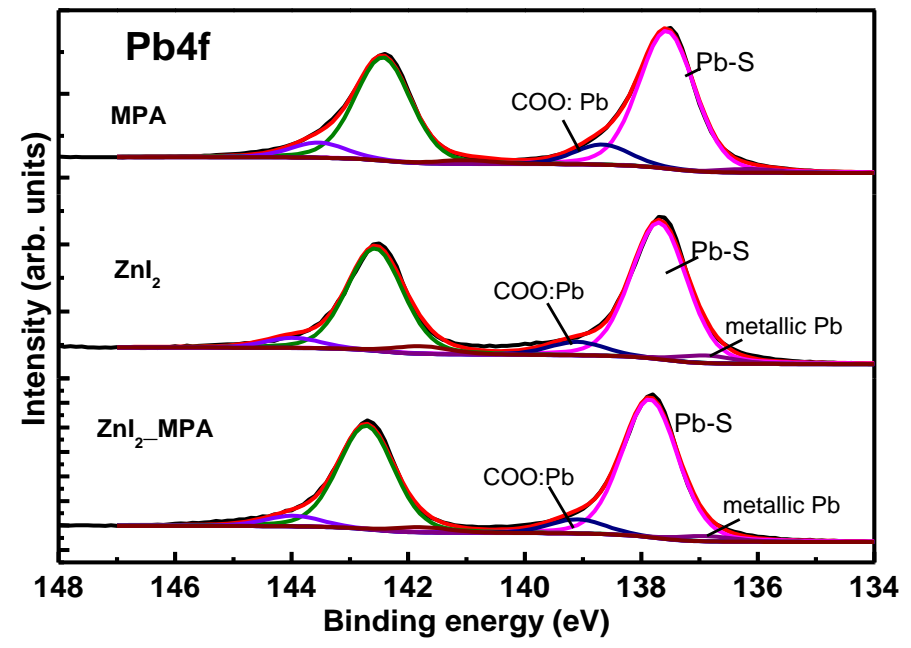

Figure 4: Deconvoluted (a) S2p, (b) O1s, (c) Pb4f XPs spectra of MPA, ZnI , and ZnI __MPA treated QD layers. With $\mathrm{ZnI}_{2}$ MPA treatment, $\mathrm{Pb}-\mathrm{OH}$, atomic $\mathrm{O}$, metallic $\mathrm{Pb}$ reduced whereas the bound thiol increased leading to better surface passivation. 

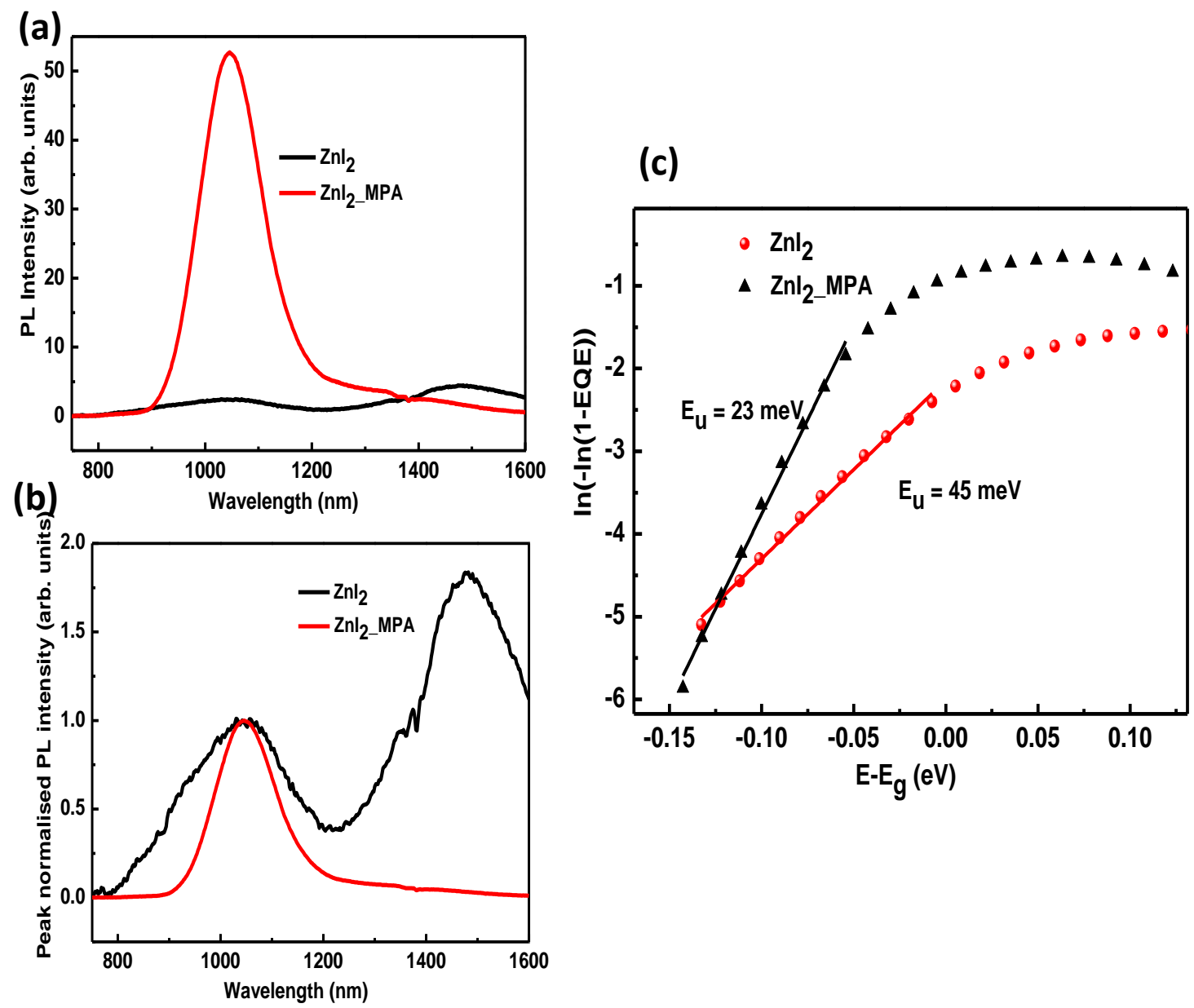

Figure 5: (a) PL intensity normalized to the absorption of the film for $\mathrm{ZnI}_{2}$ and $\mathrm{ZnI}_{2}$ MPA ligand treated films. There is a huge improvement in the band-edge emission in case of $\mathrm{ZnI}_{2} \_$MPA treated film. (b) Peak normalised PL intensity shows dominating sub-band gap emission in case of $\mathrm{ZnI}_{2}$ treated films. With MPA mixing with $\mathrm{ZnI}_{2}$ treatment, the mid-gap trap emission reduced. (c) Urbach tail energy $\left(\mathrm{E}_{\mathrm{U}}\right)$ calculated from the EQE spectrum of the PV devices. Significant $E_{U}$ reduction observed in case of mixed ligand treatment which is consistent with the PL spectra. 
TOC

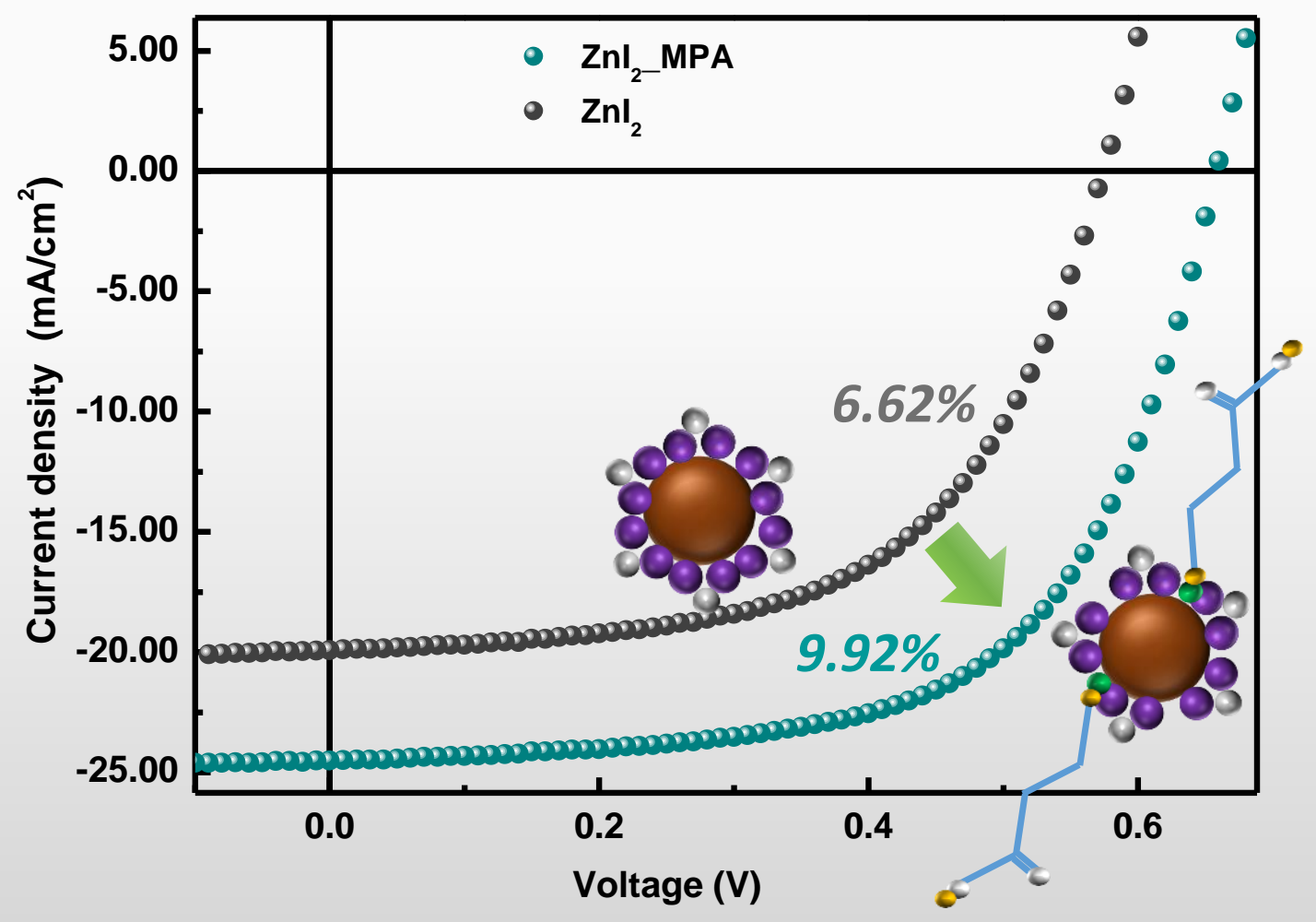

